\title{
Production des liquides de l'oreille interne
}

Les cellules sensorielles de l'oreille interne sont en contact avec deux liquides extracellulaires de composition extrêmement différente, l'endolymphe par leur pôle apical cilié et la périlymphe par leur pôle basal synaptique. La périlymphe provient du plasma au travers d'une barrière hémato-périlymphatique assez proche de la barrière hémato-encéphalique ; sa composition est voisine de celle du plasma. L'endolymphe est produite à partir de la périlymphe par des transferts trans-épithéliaux; elle a des caractères de liquide intracellulaire, riche en potassium et pauvre en sodium et est chargée positivement. Ces liquides et leurs caractéristiques de potentiel et de concentrations ioniques sont essentiels au bon fonctionnement de la transduction mécano-bioélectrique, fonction de l'oreille interne. La maladie de Ménière peut être une conséquence d'anomalies dans l'écoulement de l'endolymphe, entraînant une dilatation du labyrinthe membraneux et des modifications de composition ionique et du potentiel électrique.

Olivier Sterkers Évelyne Ferrary Patrice Tran Ba Huy

\section{ADRESSE}

O. Sterkers : professeur d'oto-rhino-laryngologie à la faculté Xavier-Bichat.

E. Ferrary : chargée de recherches Inserm.

P. Tran Ba Huy: professeur d'oto-rhinolaryngologie à la faculté Lariboisière. Département de physiologie, Inserm U. 251, faculté Xavier-Bichat, université Paris VII, 16, rue Henri-Huchard, 75018 Paris, France. 'oreille interne a pour rôle de transformer des stimuli mécaniques, tels les sons et les mouvements de la tête, en des événements électriques, ce qui permet aux vertébrés d'entendre et de maintenir leur équilibre dans l'espace. Dans l'oreille interne, deux compartiments liquidiens sont séparés par le labyrinthe membraneux, épithélium sensoriel hétérogène et serré. La lumière du labyrinthe membraneux est remplie d'endolymphe, dont la composition chimique rappelle celle d'un liquide intracellulaire mais positivement polarisé. Les espaces compris entre les labyrinthes membraneux et osseux sont remplis de périlymphe, dont la composition ressemble à celle d'un liquide extracellulaire (figure 1). Les liquides de l'oreille interne transmettent les stimuli aux organes sensoriels cochléaire et vestibulaire et participent à la transduction mécano-bioélectrique. En effet, la transduction ne peut survenir que si le pôle apical cilié des cellules sensorielles est au contact de l'endolymphe et leur pôle basal synaptique au contact de la périlymphe. S'il est bien établi que les liquides de l'oreille interne sont essentiels au fonctionnement des cellules ciliées, les lieux et mécanismes de formation de ces liquides ne sont encore qu'incomplètement élucidés.

La constatation, dans les années 50, d'un potentiel positif élevé dans l'endolymphe cochléaire et d'une concentration élevée de potassium et faible de sodium dans l'endolymphe a stimulé les recherches sur les liqui- 
des de l'oreille interne. L'application à l'oreille interne des techniques développées pour l'étude d'autres épithéliums est difficile à cause de la petite taille de l'organe et de sa situation profonde dans l'os. Le développement récent de techniques in vitro a cependant permis de réaliser des progrès dans la compréhension de la production des liquides de l'oreille interne.

\section{Origines de la périlymphe}

La composition de la périlymphe qui baigne la face externe (abluminale) de l'épithélium labyrinthique n'est pas uniforme dans la cochlée et diffère selon que la périlymphe est prélevée de la scala vestibuli ou de la scala tympani (figure 1). Les concentrations de potassium, glucose et protéines dans la périlymphe de la scala vestibuli (périlymphe vestibulaire) sont supérieures à celle de la périlymphe de la scala tympani (périlymphe tympanique). Ces observations ont suggéré que l'origine des périlymphes des deux rampes était différente. Des études cinétiques ont montré que l'origine de la périlymphe vestibulaire est essentiellement le plasma alors que celle de la périlymphe tympanique reste discutée chez les rongeurs. En effet, le mannitol et le sucrose injectés dans le plasma pénètrent plus lentement dans la périlymphe tympanique et le liquide céphalorachidien (LCR) que dans la périlymphe vestibulaire. Les mêmes traceurs, injectés dans le ventricule cérébral latéral, c'est-à-dire dans le LCR, sont retrouvés essentiellement dans la périlymphe tympanique (figure 2, [1]). Le fait qu'aucune radioactivité n'est détectée dans la périlymphe tympanique lorsqu'une solution d'électrolytes radioactifs est perfusée dans la périlymphe vestibulaire en même temps qu'une solution non marquée dans la périlymphe tympanique $[2,3]$ suggère que les sources des deux périlymphes sont indépendantes.

Des arguments physiologiques et morphologiques ont établi l'existence d'une barrière entre le plasma et la périlymphe vestibulaire qui a de nombreux points communs avec la barrière hémato-encéphalique. La barrière hémato-périlymphatique restreint l'entrée des électrolytes et des

\section{* GLOSSAIRE *}

Cochlée ou labyrinthe antérieur: structure contenant l'organe sensoriel responsable de l'audition.

Vestibule ou labyrinthe postérieur: structure contenant les organes sensoriels de l'équilibration qui sont les macules de l'utricule et du saccule et les crêtes ampullaires des trois canaux semi-circulaires.

Organe de Corti : organe sensoriel de l'audition. Il comprend les cellules ciliées internes et externes et leurs cellules de soutien.

Strie vasculaire : structure épithéliale cochléaire, située dans le mur latéral de la cochlée, responsable de la sécrétion de l'endolymphe. Elle est formée de plusieurs couches cellulaires: les cellules marginales en regard de l'endolymphe, les cellules intermédiaires et le réseau capillaire de la strie, les cellules basales qui reposent sur le ligament spiral en rapport avec les rampes périlymphatiques.

Membrane de Reissner: structure épithéliale cochléaire formée d'une assise de cellules épithéliales unies par des jonctions serrées et reposant sur une couche cellulaire mésothéliale lâche en regard de la rampe périlymphatique vestibulaire.

Cellules marginales : cellules de la strie vasculaire présumées responsables de la sécrétion de l'endolymphe. Leur pôle apical est en contact avec l'endolymphe. Les replis de leur membrane basolatérale contiennent de nombreuses mitochondries et sont le siège d'une activité élevée en $\mathrm{Na}^{+}$, $K^{+}-A T P a s e$

Cellules sombres: cellules du vestibule présumées responsables de la sécrétion de l'endolymphe et situées dans l'utricule et les ampoules des canaux semi-circulaires. petites molécules neutres dans la périlymphe vestibulaire selon l'ordre suivant (Tableau I, p. 758) : Na $\sim \mathrm{K}$ $\sim \mathrm{Cl} \sim$ D-glucose $>$ urée $>$ Lglucose $\sim$ mannitol $\sim$ sucrose. Seules les petites molécules hydrosolubles entrent dans la périlymphe vestibulaire à travers la barrière hémato-périlymphatique, en fonction de leur poids moléculaire, exception faite du D-glucose et du glycérol. Le D-glucose pénètre dans la périlymphe vestibulaire par l'intermédiaire d'un transport facilité. Il a été démontré que ce transport est stéréospécifique et saturable, et que le glucose et ses analogues structuraux sont en compétition vis-à-vis du transporteur; mais le transport n'a pu être inhibé in vivo [4].

A l'inverse de ce qui est observé à travers la barrière hémato-encéphalique, le sodium et le chlore pénètrent plus rapidement que l'urée dans la périlymphe vestibulaire. La formation de la périlymphe vestibulaire résulterait de ce fait de l'association de plusieurs mécanismes. De nombreux arguments en faveur d'une sécrétion épithéliale de la périlymphe vestibulaire proviennent des similitudes de cette sécrétion avec celle du LCR par le plexus choroïde : 1) les valeurs du rapport des constantes de transfert sodium/urée sont semblables dans la périlymphe vestibulaire et le LCR ; 2) l'administration intraveineuse d'acétazolamide, qui inhibe l'anhydrase carbonique, entraîne une diminution de l'entrée du chlore dans la périlymphe vestibulaire et le LCR [5] ; 3) dans la zone vasculoépithéliale du limbus spiral, une activité élevée d'anhydrase carbonique et de $\mathrm{Na}^{+}, \mathrm{K}^{+}$-ATPase a été identifiée, ce qui indique un équipement enzymatique comparable à celui du plexus choroïde. Ainsi la périlymphe vestibulaire serait formée à partir du plasma par deux mécanismes au moins : des échanges de substances dissoutes à travers une barrière hémato-périlymphatique et une sécrétion épithéliale probablement localisée dans le limbus spiral. Le rôle respectif de ces deux mécanismes mériterait d'être défini lorsque l'on sait que la périlymphe vestibulaire représente la voie principale d'accès à l'espace endolymphatique [6].

L'origine de la périlymphe tympani- 


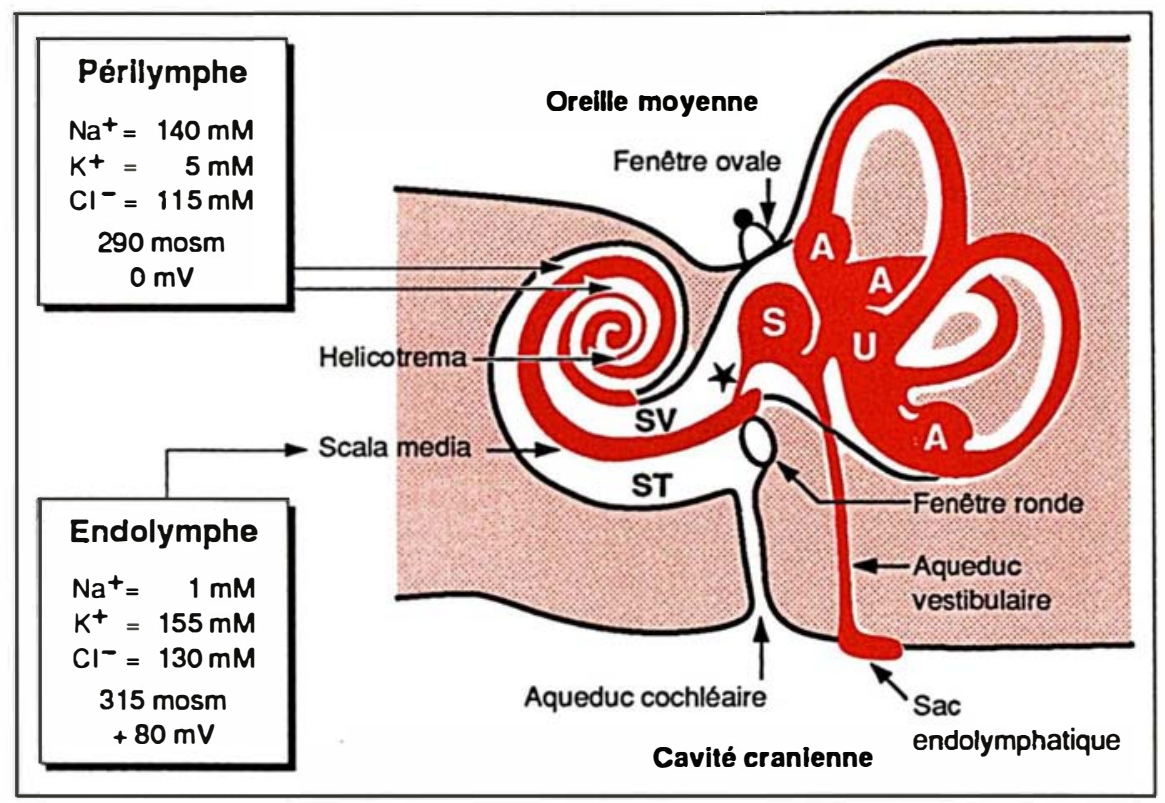

Figure 1. Le labyrinthe membraneux (en rouge) est composé de cavités qui communiquent entre elles. Elles sont incluses dans le labyrinthe osseux (en rose). La lumière de ces cavités est remplie d'endolymphe, un liquide riche en $K$, positivement polarisé. Les espaces compris entre le labyrinthe membraneux et le labyrinthe osseux (en gris) contiennent la périlymphe, dont la composition ressemble à celle d'un liquide extracellulaire. A : ampoule des canaux semicirculaires, $S$ : saccule, ST: scala tympani, $S V$ : scala vestibuli, $U$ : utricule; $\star$ : canal de Hensen. (Adapté de [20]).

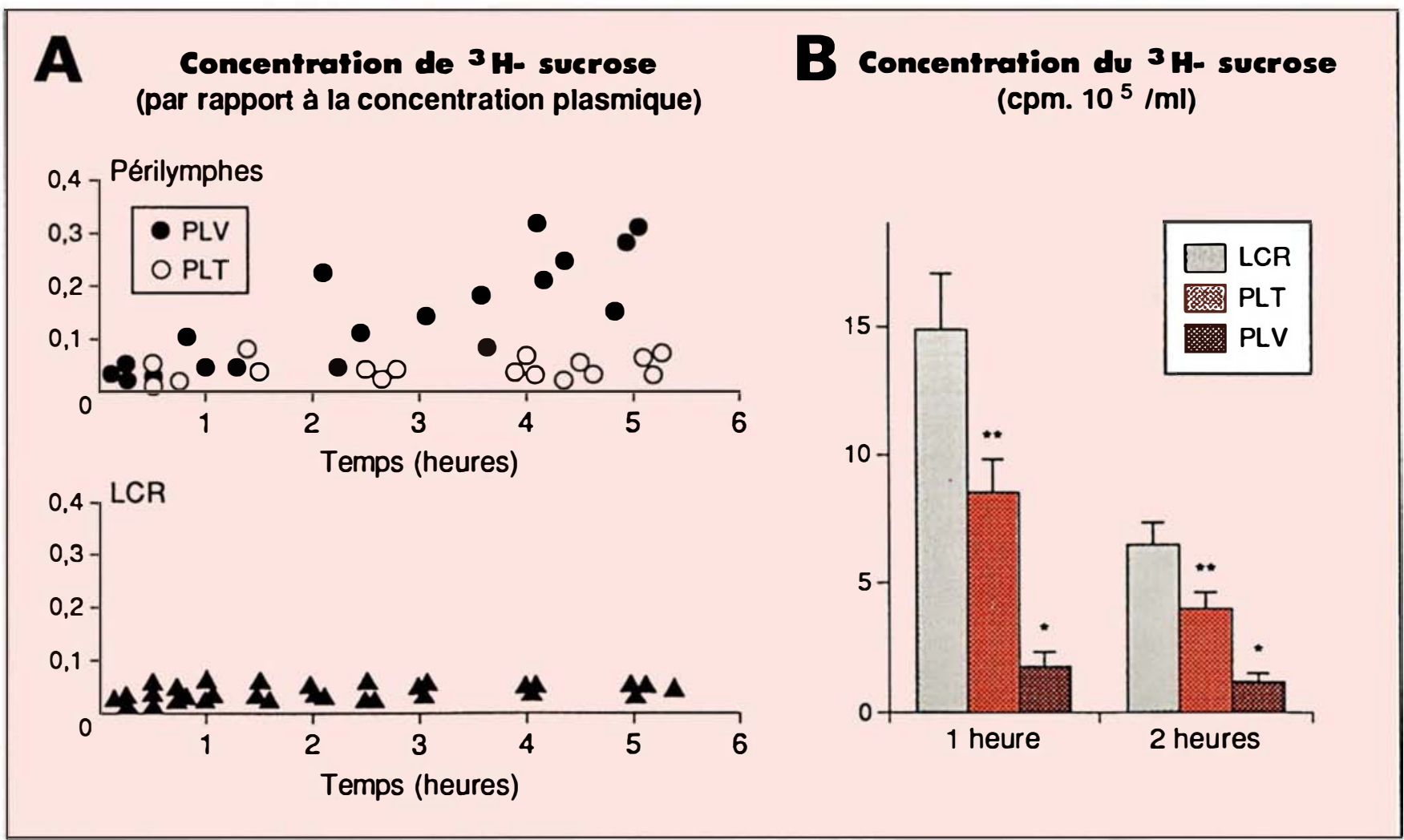

Figure 2. A. Entrée de sucrose radioactif dans la périlymphe de la rampe vestibulaire (PLV), la périlymphe de la rampe tympanique (PLT) et le liquide céphalorachidien (LCR) après I'administration intraveineuse du traceur. B. Concentration de sucrose radioactif dans le LCR, la PLV et la PLT une et deux heures après une injection de $2 \mu \mathrm{l}$ de la solution de traceur dans le ventricule cérébral latéral. Chaque colonne est la moyenne de 4 valeurs. La valeur dans la PLT est inférieure à celle du LCR (* "P $<0,05$ ) et la valeur dans la PLV est inférieure à celle de la PLT ("P $<0,05$ ) et représente environ $10 \%$ de la valeur dans le LCR. (D'après [9]). 
Tableau

CONSTANTES D'ÉCHANGES (k entrée, min-1) DES ÉLECTROLYTES

ET DES SUBSTANCES DISSOUTES NEUTRES A TRAVERS LA BARRIĖRE HÉMATO-PÉRILYMPHATIQUE (ÉCHANGES ENTRE LE PLASMA ET

LA PÉRILYMPHE VESTIBULAIRE) ET A TRAVERS LA BARRIĖRE ÉPITHÉLIALE (ÉCHANGES ENTRE LA PÉRILYMPHE ET L'ENDOLYMPHE) (d'après [1, 4-6])

\section{RÉFÉRENCES}

1. Sterkers O, Ferrary E, Saumon G, Amiel $\mathrm{C}$. $\mathrm{Na}$ and non electrolyte entry into inner ear fluids of the rat. Am J Physiol $1987 ; 253: 50-8$.

2. Konishi T, Hamrick PE, Walsh PJ. Ion transport in guinea pig cochlea. I. K and $\mathrm{Na}$ transport. Acta Otolaryngol $1978 ; 86$ : 22-34

3. Konishi T, Hamrick PE. Ion transport in the cochlea of guinea pig. II. Chloride transport. Acta Otolaryngol $1978 ; 86$ : 176-84.

4. Ferrary E, Sterkers O, Saumon G, Tran Ba Huy P, Amiel C. Facilitated transfer of glucose from blood into perilymph in the rat cochlea. Am J Physiol 1987; 253: 59-65.

5. Sterkers O, Saumon G, Tran Ba Huy P, Ferrary E, Amiel C. Electrochemical heterogeneity of the cochlear endolymph : effect of acetazolamide. Am J Physiol 1984; 246 : 47-53.

6. Sterkers O, Saumon G, Tran Ba Huy P, Amiel C. $\mathrm{K}, \mathrm{Cl}$, and $\mathrm{H}_{2} \mathrm{O}$ entry in endolymph, perilymph, and cerebrospinal fluid of the rat. Am J Physiol $1982 ; 243$ : 173-80.

7. Sterkers O, Ferrary E, Amiel C. Production of inner ear fluids. Physiol Rev $1988 ; 68: 1083-128$

8. Hara A, Salt AN, Thalmann R. Perilymph composition in scala tympani of the cochlea : influence of cerebrospinal fluid. Hearing Res 1989; 42 : 265-72.

9. Oudar O, Ferrary E, Feldmann G. Ultrastructural study of the semicircular canal cells of the frog Rana esculenta. Anat $\operatorname{Rec} 1988 ; 220: 328-34$

10. Marcus NY, Marcus DC. Potassium secretion by non sensory region in gerbil utricle in vitro. Am J Physiol 1987; 253 : 613-21.

11. Marcus DC, Marcus NY, Greger R Sidedness of action of loop diuretics and ouabain on non sensory cells of utricle : A micro-Ussing chamber for inner ear tissues.

que est discutée. La scala tympani communique en effet à la fois avec la scala vestibuli par l'helicotrema, à l'apex de la cochlée, et avec la grande citerne cérébelleuse par l'aqueduc cochléaire, à la base (figure 1). Ainsi la périlymphe tympanique est habituellement considérée comme un liquide de transition, intermédiaire entre la périlymphe vestibulaire et le LCR. Cependant, les études sur la périlymphe tympanique n'ont été réalisées qu'à la base de la cochlée où les prélèvements de périlymphe sont aisés. Chez les rongeurs dont l'aqueduc cochléaire est largement perméable, les études ont été réalisées après le blocage de l'aqueduc cochléaire ou après ouverture de la citerne. Ces travaux n'ont pas apporté d'arguments concluants (pour revue voir [7]). Néanmoins, le fait que la concentration de glycine dans la périlymphe tympanique soit voisine de celle de la périlymphe vestibulaire lorsque le volume de périlymphe prélevé est inférieur à $200 \mathrm{nl}$, alors qu'elle se rapproche de celle du LCR pour des volumes supérieurs, suggère que la périlymphe tympanique est formée localement sans échanges majeurs avec le LCR [8].

\section{Sécrétion de l'endolymphe}

L'endolymphe cochléaire est caracté- risée par des concentrations élevée de potassium $(150 \mathrm{mM})$ et faible de sodium (1 $\mathrm{mM}$ ), une osmolalité élevée (supérieure de 10 à 40 mos$\mathrm{mol} / \mathrm{kg} \mathrm{H}_{2} \mathrm{O}$ à celles de la périlymphe et du plasma) et un potentiel transépithélial positif. Un rapport potassium/sodium tout à fait inhabituel est retrouvé dans les différentes cavités de l'oreille interne des vertébrés. La valeur du potentiel endolymphatique varie en revanche de $+100 \mathrm{mV}$ dans la cochlée à $+5 \mathrm{mV}$ dans le vestibule ou l'oreille interne des vertébrés inférieurs. Deux faits sont bien établis : d'une part, l'endolymphe est formée à partir de la périlymphe et non du plasma par les échanges transépithéliaux de substances dissoutes (Tableau I); d'autre part, la $\mathrm{Na}^{+}, \mathrm{K}^{+}$-ATPase fournit l'énergie nécessaire à ces échanges. L'identification de la périlymphe comme compartiment précurseur de l'endolymphe a été obtenue par l'analyse cinétique de pénétration du potassium et du chlore dans l'endolymphe après injection de leurs traceurs radioactifs dans le plasma ou la périlymphe $[2,3,6]$. La mise en évidence du rôle de la $\mathrm{Na}^{+}$, $\mathrm{K}^{+}$-ATPase dans la formation de l'endolymphe (pour revue voir [7]) repose sur les arguments suivants: 1) une activité élevée de cette enzyme existe dans les cellules mar- 
ginales de la strie vasculaire et dans les cellules sombres vestibulaires, les cellules de ces deux structures étant responsables de la sécrétion de l'endolymphe ; 2) le potentiel endolymphatique et le gradient de sodium et de potassium entre l'endolymphe et la périlymphe sont abolis par l'administration périlymphatique d'ouabaïne ou la suppression du potassium de la périlymphe ; 3) l'activité de la $\mathrm{Na}^{+}, \mathrm{K}^{+}$-ATPase dans la strie vasculaire et les valeurs du potentiel endocochléaire et des concentrations de potassium et de chlore varient parallèlement le long de la cochlée suivant un gradient qui croît de l'apex vers la base.

Les différences de composition de l'endolymphe dans les différentes cavités de l'oreille interne peuvent découler de deux phénomènes : premièrement, les tissus de l'oreille interne ont des capacités de transport transépithélial inégales. Si la strie vasculaire et les cellules sombres apparaissent comme spécialisées dans les transports actifs ioniques, les autres structures - comme la membrane de Reissner, la partie non ampullaire du canal et les parois indifférenciées de l'utricule et du saccule - ne semblent pas avoir de propriété sécrétrice importante ; deuxièmement, les structures sécrétrices de l'endolymphe, la strie vasculaire et les cellules sombres, diffèrent par leur structure et leur situation dans chacune des cavités labyrinthiques. Les cellules sombres, dont la morphologie est comparable à celle des cellules marginales de la strie, comprennent une seule assise cellulaire alors que la strie vasculaire est formée, outre les cellules marginales, des cellules intermédiaires, de capillaires et d'une assise de cellules basales. De plus, la strie vasculaire occupe le mur latéral de la cochlée de façon ininterrompue de l'apex à la base de la cochlée, tandis que les cellules sombres sont localisées dans des aires relativement petites des ampoules des canaux semi-circulaires et de l'utricule [9].

L'existence d'une pompe électrogène à potassium qui serait comparable à la fois au potentiel endocochléaire et à la concentration élevée de potassium dans l'endolymphe est actuellement controversée. Cette pompe, qui siègerait dans la membrane luminale des cellules marginales de la strie vasculaire, engendrerait un potentiel positif de $120 \mathrm{mV}$. Le potentiel de + $80 \mathrm{mV}$ mesuré dans l'endolymphe résulterait de la somme algébrique de ce potentiel électrogène de potassium et d'un potentiel de diffusion du potassium ( $-40 \mathrm{mV}$ ) qui est très vraisemblablement créé par la fuite de potassium à travers les cellules ciliées de l'organe de Corti. Si l'existence du potentiel de diffusion est démontrée, l'hypothèse d'une pompe électrogène à potassium ne repose que sur l'argument suivant: le potentiel endocochléaire et le transport actif de potassium récupèrent parallèlement après leur inhibition par l'administration intraveineuse d'acide éthacrynique $(60 \mathrm{mg} / \mathrm{kg})$ ou de furosémide (100 mg/kg). Remarquons qu'une pompe électrogène à potassium n'a jamais été décrite dans un épithélium de mammifere, alors qu'il existe dans des épithéliums d'insectes de telles pompes insensibles à l'ouabaïne mais responsables de gradients osmotiques locaux.

Les études menées in vitro sur des préparations isolées d'utricule de mammifère $[10,11]$, de canal semicirculaire de batracien [12] et poisson [13] ont permis de définir et de localiser un certain nombre de transporteurs ioniques dans les cellules sécrétrices (figure 3). Jusqu'à présent, il a ainsi été possible d'identifier : dans la membrane basolatérale, une $\mathrm{Na}^{+}$, $\mathrm{K}^{+}$-ATPase sensible à l'ouabaïne et un cotransport $\mathrm{Na}+-\mathrm{K}+-\mathrm{Cl}-$ sensible aux diurétiques de l'anse (furosémide, bumétanide); dans la membrane luminale, un canal sodique responsable de $60 \%$ de la réabsorption du sodium endolymphatique. Le système responsable de la sécrétion luminale du potassium reste inconnu : il ne s'agit ni d'un canal potassique, ni d'un cotransport $\mathrm{Na}+-\mathrm{K}+-\mathrm{Cl}-$ sensible aux diurétiques. De plus, l'observation récente

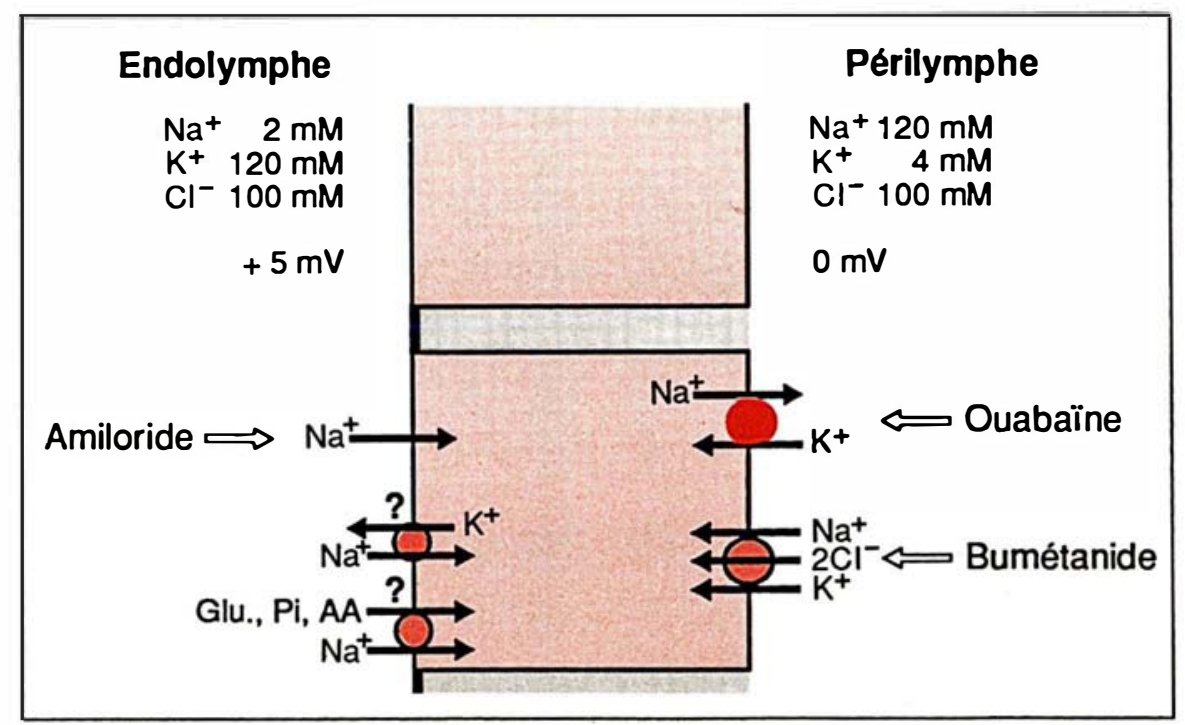

Figure 3. Modèle de sécrétion de l'endolymphe par une cellule sombre de l'épithélium du canal semi-circulaire de grenouille. Les différents transports identifiés et leurs inhibiteurs spécifiques sont indiqués sur les membranes luminale (endolymphatique) et basolatérale (périlymphatique). Le mode de sécrétion luminale de $\mathrm{K}_{1}^{+}$reste hypothétique. $\rightarrow$ diffusion, P-transport actif, ШCo-transport, $\longrightarrow$ contre-transport, Glu : glucose ; AA : acides aminés ; Pi : phosphate. (Adapté de [12]). 


\section{RÉFÉRENCES}

12. Ferrary E, Bernard C, Sterkers O, Amiel C. Sodium transfer from endolymph through a luminal amiloride-sensitive channel. Am J Physiol 1989; 257 : 182-9.

13. Garvin JL, Spring KR, Santi PA. Secretion of endolymph by semicircular canals of the shark. Am J Physiol 1988 ; 255: 711-9.

14. Ikeda K, Morizono T. Electrochemical profiles for monovalent ions in the stria vascularis : Cellular model of ion transport mechanisms. Hearing Res 1989 ; 39 : 279-86.

15. Shinozaki N, Kimura RS. Scanning electron microscopic observations on the distended Reissner's and saccular membranes in the guinea pig. Acta Otolaryngol 1980 ; $90: 370-84$.

16. Horner KC, Cazals Y. Rapidly fluctuating thresholds at the onset of experimentally-induced hydrops in the guinea pig. Hearing Res 1987 ; 26 : 319-25.

17. Horner KC, Guilhaume A, Cazals Y. Atrophy of middle and short stereocilia on outer hair cells of the guinea pig cochleas with experimentally induced hydrops. Hearing Res $1988 ; 32: 41-8$.

18. Sziklai I, Horner KC, Ferrary E, Sterkers O, Amiel C. Electrochemical composition of the cochlear fluids in the early experimental hydrops. Acta Otolaryngol 1989; $107: 371-4$.

19. Sziklai I, Ferrary E, Horner KC, Sterkers O, Amiel C. Time-related alteration of endolymph composition in an experimental model of endolymphatic hydrops. Version révisée soumise pour publication.

20. Sterkers O, Ferrary E, Amiel C. How are the inner ear fluids formed? News In d'un potentiel intracellulaire positif dans les cellules marginales [14] complique plus qu'elle ne simplifie la compréhension des phénomènes impliqués dans la sécrétion de l'endolymphe. Il n'existe pas, à notre connaissance, d'autres cellules chargées positivement de manière constante. Comment fonctionne une cellule chargée à $+100 \mathrm{mV}$ est le premier élément à élucider avant de découvrir le secret de la sécrétion de potassium. Il est actuellement impossible d'expliquer la genèse du potentiel endocochléaire et la sécrétion de potassium de l'endolymphe par le seul fonctionnement de la cellule marginale. D'autres hypothèses que la pompe électrogène à potassium ne peuvent être écartées, comme, par exemple, celle d'une rétrodiffusion de sodium dans l'endolymphe par les voies paracellulaires de la strie ou/et une perméabilité au chlore supérieure à celle du potassium de la membrane de Reissner.

La perméabilité à l'eau de l'épithélium cochléaire quelle que soit la structure concernée (strie vasculaire, membrane de Reissner, organe de Corti) doit être faible. Cela est nécessaire pour rendre compte de l'hyperosmolalité de l'endolymphe par rapport à la périlymphe et du maintien du gradient osmotique dans l'endolymphe. L'homéostasie de l'endolymphe est assurée par des échanges ioniques radiaires transépithéliaux vraisemblablement non couplés à des mouvements d'eau, et d'un flux d'eau longitudinal nécessairement créé par le gradient osmotique qui existe entre l'apex et la base de la cochlée. A la base de la cochlée, le canalis reuniens de Hensen permet l'écoulement de ce flot longitudinal vers le saccule et le sac endolymphatique (figure 1). De nombreux arguments sont en faveur d'un rôle non négligeable du sac endolymphatique dans la réabsorption de l'endolymphe (pour revue voir [7]).

\section{Modèle \\ physiopathologique de la maladie de Ménière : I'hydrops endolymphatique expérimental}

La destruction du sac endolymphatique provoque un hydrops endolym- phatique dans plusieurs espèces étudiées (constant chez le cobaye et le lapin). Cet hydrops est remarquable par la distension de la membrane de Reissner qui est déjà visible 24 heures après la destruction du sac [15]. $\mathrm{Au}$ cours de l'hydrops endolymphatique expérimental, des modifications de pression hydrostatique dans la cochlée retentissent à la fois sur l'organe de Corti, ce qui entraîne une atteinte auditive, et sur la membrane de Reissner, qui se distend progressivement. L'effet de l'hydrops sur l'audition [16], les cellules sensorielles [17] et la composition électrochimique de l'endolymphe $[18,19]$ a montré que l'évolution de l'hydrops se fait en trois étapes successives. La première, qui dure plus de 2 semaines et moins de 6 semaines, est une étape à pression apparemment normale. L'hyperpression qui résulte de l'interruption du flot d'endolymphe vers le sac est immédiatement amortie du fait de la compliance de la membrane de Reissner. Il s'ensuit une distension progressive de la membrane de Reissner, une surdité fluctuante sur les fréquences graves, tandis que la composition électrochimique de l'endolymphe est conservée. La deuxième étape est observée entre 2 et 6 semaines jusqu'à plus de 9 semaines. C'est un stade d'hyperpression avec distension progressive de la membrane de Reissner. La pression augmente dans le canal cochléaire en raison de la persistance de la sécrétion endolymphatique et de la suppression de l'issue d'endolymphe vers le sac endolymphatique. Cependant, l'augmentation de la pression est de moins en moins amortie par la membrane de Reissner de plus en plus distendue. La surdité sur les fréquences graves est stable et celle sur les fréquences aiguës apparaît. L'endolymphe a une composition électrochimique uniforme le long de la cochlée : les gradients baso-apicaux sont dissipés et l'osmolalité est diminuée quoique supérieure à celle de la périlymphe.

La troisième étape débute après la $9^{e}$ semaine. C'est un stade d'hyperpression avec distension maximale de la membrane de Reissner. Il existe une surdité en plateau stable; le potentiel endocochléaire reste diminué mais positif ; les gradients électrochi- 
miques baso-apicaux sont toujours dissipés, alors que l'endolymphe est à nouveau concentrée. La persistance d'un liquide riche en potassium, dépourvu de sodium, hyperosmotique et encore positivement polarisé témoigne du maintien de la sécrétion de l'endolymphe, alors que les modifications de la composition électrochimique de l'endolymphe suggèrent que les perméabilités aux $\mathrm{K}^{+}$et $\mathrm{Cl}-$ de la membrane de Reissner sont affectées.

\section{Conclusion}

L'étude des liquides de l'oreille interne a permis d'identifier des mécanismes de sécrétion faisant appel à une barrière hémato-périlymphatique responsable de la formation de la périlymphe et une barrière épithéliale qui sécrète l'endolymphe. La barrière hémato-périlymphatique présente des similitudes avec à la fois la barrière hémato-encéphalique et la barrière hémato-céphalorachidienne. Le labyrinthe membraneux par l'intermédaire duquel l'endolymphe est sécrétée est le siège de transports similaires à ceux rencontrés dans des épithéliums de type serré tels le tube distal du rein, la vessie ou la peau du batracien. Dans le labyrinthe membraneux, l'étape cellulaire a été franchie pour l'étude des cellules sensorielles mais pas encore pour celle des cellules sécrétrices. Le rôle de l'épithélium non sécrétoire dans l'homéostasie de l'endolymphe, qui a été négligé pendant longtemps, a été mis en évidence par les modifications de l'endolymphe au cours de l'hydrops endolymphatique expérimental

\section{Summary}

Production of the inner ear fluids

The inner ear fluids differ widely in electrochemical composition and origin. The perilymph resembles an extracellular fluid whereas endolymph is a $\mathrm{K}+$-rich, almost $\mathrm{Na}+$-free, positively polarized fluid. The perilymph of scala vestibuli originates mainly from plasma across a blood-perilymph barrier that restricts the entry of small hydrophilic solutes except glucose which is transferred into perilymph by a facilitated transport. The blood-perilymph barrier is most likely composed of an endothelial barrier associated with an epithelial secretion. The latter could be located at the vasculoepithelial zone of the spiral limbus. The formation of endolymph proceeds from perilymph. The active secretion of endolymph is localized in the stria vascularis in the cochlea and in the dark cells in the vestibule. The basolateral $\mathrm{Na}^{+}$, $\mathrm{K}^{+}-\mathrm{ATPase}$ and a $\mathrm{Na}{ }^{+}-\mathrm{K}+$ $\mathrm{Cl}-$ cotransport are involved in transepithelial potential generation and in the chemical formation of endolymph. Part of $\mathrm{Na}^{+}$is transferred out of endolymph through an apical amiloride-sensitive $\mathrm{Na}+$ channel along an electrochemical gradient provided by the activity of the basolateral $\mathrm{Na}^{+}$, $\mathrm{K}^{+}$-ATPase. The role of the non-sensory, non-secretory parts of the labyrinthine epithelium, suggested by the alterations of endolymph during endolymphatic hydrops, remains to be elucidated. In vitro preparations of inner ear epithelium have been set up and are promising tools for inner ear fluid studies.

\section{Remerciements}

Les auteurs remercient le professeur Claude Amiel pour ses conseils permanents dans l'élaboration de ces travaux et pour ses critiques constructives dans la rédaction du manuscrit. Ils remercient également Françoise Carlier pour la préparation du manuscrit.

\section{TIRÉS A PART}

O. Sterkers. 